\title{
HYBRID INDOOR TRACKING OF HUMANS IN HAZARDOUS ENVIRONMENTS
}

\author{
Andreas Fink, Helmut Beikirch \\ University of Rostock, Department of Computer Science and Electrical Engineering \\ A.-Einstein-Str. 2, D-18059 Rostock, Germany \\ \{andreas.fink;helmut.beikirch\}@uni-rostock.de \\ www.igs.uni-rostock.de
}

\begin{abstract}
The reliable tracking of humans and materials in indoor scenarios is an ongoing research issue. For example, the monitoring of humans in partially hazardous environments - like the surroundings of an underground longwall mining infrastructure - is crucial to save human lives. A centroid location estimation technique based on received signal strength (RSS) readings offers a well known and low-cost tracking solution in such a rough environment where many other systems with optical, magnetical or ultrasound sensors fail. Due to signal fading the RSS values alone cannot ensure a precise tracking. The sensor fusion of the RSS-based localization with an inertial navigation system (INS) leads to a more precise tracking. The long-term stability of the RSS-based localization and the good shortterm accuracy of the INS are combined using a Kalman filter. The experimental results on a motion test track show that a tracking of humans in multipath environments is possible with low infrastructural costs.
\end{abstract}

Keywords: Inertial Navigation System, Kalman Filter, Received Signal Strength, Indoor Tracking, Sensor Fusion.

\section{INTRODUCTION}

In an environment with partially dangerous areas a rough but high-available localization of humans with a monitoring of their movement patterns is of interest. For example, the detection of maintenance staff in the longwall mining, in particular close to self-advancing hydraulic shields is necessary.

Longwall mining is a highly productive underground coal mining technique where massive shearers cut coal from a wall face, which falls onto a conveyor belt for removal. The hydraulic shields are placed in a long line in order to support the roof of the coalface and maintaining a safe working space along the face for the miners. The automatic motion of the hydraulic shields needs to be stopped when a miner is localized in front of them. The accuracy of the localization system determines the size of the security zone and the amount of shields which are involved. Besides the accuracy also the availability of the localization system plays a significant role for the availability and efficiency of the longwall mining system, since the safety critical system architecture requires an immediate idle state of the system.

The environment of a longwall mining system does not permit a global radio-based positioning (e.g. GPS). A local radio-based system with a multilateration of several distance approximations between an unknown node (blind node, BN) and fixed anchor nodes (reference nodes, RNs) is applicable to find out the position of a miner equipped with a BN.

The received signal strength (RSS) offers a lowcost sensor for ranging-based localization systems. A classification of the ranging-based techniques is shown in Fig. 1. The use of directional sensors like infra-red (IR), ultrasound, optical and magnetic systems is limited to line-of sight (LOS) scenarios. There are several RF-based methods which can be used also in non-line-of sight (NLOS) scenarios. In principle the location estimation can be realized via analyzing signal propagation delays (time of arrival TOA, time difference of arrival - TDOA), receive directions (Angle of Arrival - AOA) or RSS readings.

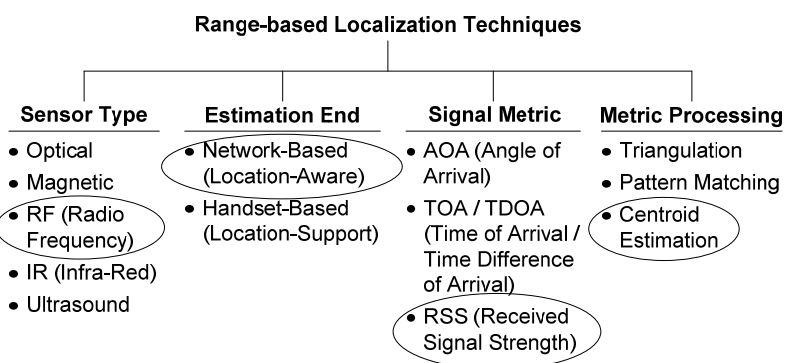

Fig. 1 - Classification of range-based localization techniques. Source: [1] 
The methods are more or less affected by the problems of multipath propagation and resulting fading effects. For multipath environments the distances based on RSS measurements are often erroneous, especially for dynamic environments where the position of obstacles (or humans) may change continuously. Thus, for a range-based localization it is difficult to rely only on the raw RSS values. For a correct interpretation of the values according to a path loss model additional measures are required, since the influence of large-scale and small-scale fading effects leads to bad distance estimations. [2],[3]

The small-scale fading due to reflection, diffraction and scattering on obstacles is the main issue. When the transmitter or receiver change their position by small movements in the order of the wavelength (e.g. $\approx 12.5 \mathrm{~cm}$ for $2.4 \mathrm{GHz}$ ) the RSS may vary by three or four orders of magnitude $(30 . .40 \mathrm{dBm})$. Therefore it is challenging to approximate the distance between transmitter and receiver with the RSS only. A detailed description of fading principles is given in [4], [5] and [6].

With a combining of the information coming from different types of sensors the accuracy of the localization can be improved. Compared to a system relying on a single sensor, the location estimation has a reduced uncertainty and an increased tolerance to single point failures. It is a common used technique to combine the position estimations of an RF-based system with an inertial navigation system (INS) [7], [8], [9]. In comparison to the RSS-based RF positioning system no external references are necessary for the INS.

With the acceleration information of the $\mathrm{BN}$ it is possible to calculate the change in velocity and position by successive mathematical integration of the acceleration with respect to time. The rotational motion of the $\mathrm{BN}$ according to the environment (reference frame) must also be taken into account so that a three-dimensional INS overall has six degrees of freedom (DOF). The rotations around the acceleration directions may be sensed using gyroscopic sensors. The latest advances in microelectro-mechanical (MEMS) acceleration and gyroscopic sensors support the design of a low-cost inertial measurement unit (IMU). Sometimes an additional earth magnetic field sensor and a preasure sensor are added to the IMU to support the heading and height calculation in the three-dimensional space.

In this paper we propose the sensor fusion of a proprietary RF localization system and a low-cost INS using a Kalman filter. In section II, the infrastructure components and algorithms of the RF localization system are described together with the challenging multipath fading. In section III, the INS with the system architecture of the low-cost IMU is presented. The procedure of the sensor fusion with a Kalman filter is given in section IV. In section $V$, we validate the localization system performance by experimental results of a dynamic measurement on a motion test track. In the last section VI, the results are discussed and investigated in terms of an outlook for further system developments.

\section{RF LOCALIZATION SYSTEM}

The infrastructure components of the RF localization system are shown in Fig. 2. We assume a one-dimensional localization scenario as it is given in an underground longwall mining application [10], although the system is also applicable for a twodimensional localization (e.g. in a factory building) [11].

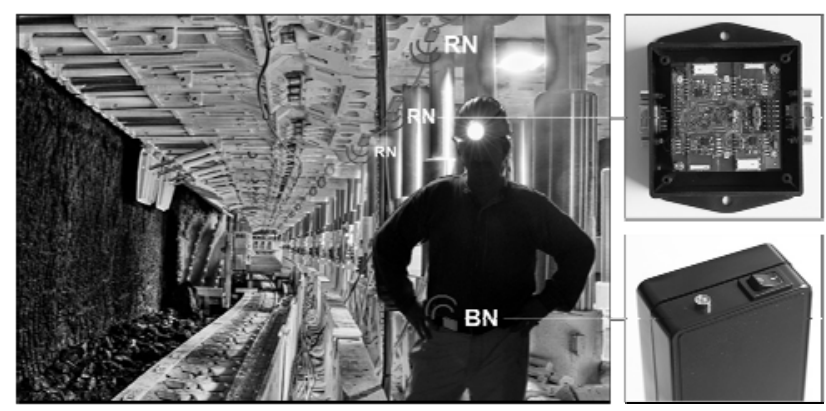

Fig. 2 - RF localization system with infrastructure components in an underground longwall coal mining tracking application ( $\mathrm{RN}$ - reference node, $\mathrm{BN}$ - blind node). Source: Own elaboration

The blind node (BN) which should be located can move in front of a line of reference nodes (RNs) with fixed positions. The RNs are connected to a date concentrator (e.g. industrial PC) via a wired bus interface (e.g. CAN). The localization process can be subdivided into the components data acquisition, data preprocessing and location estimation (cf. Fig. 3 ). The single processing steps are described briefly in the following.

- In the data acquisition phase the RNs receive packets from the $\mathrm{BN}$ and store the corresponding RSS values. Since we use a multimodal diversity platform with four single $\mathrm{RF}$ transceivers on the $\mathrm{BN}$ and the RNs more than one packet can be received at the $\mathrm{RN}$. The redundant values are transmitted to the data concentrator using the CAN bus.

- In the data preprocessing phase a selection combining (SC) of the redundant RSS values is done. The maximum RSS of each RN is computed and transformed into a weight.

- In the location estimation phase the BN position is calculated with the weighted centroid of the known RN positions. A detailed description of the used selective 
adaptive weighted centroid location estimation algorithm can be found in [10].

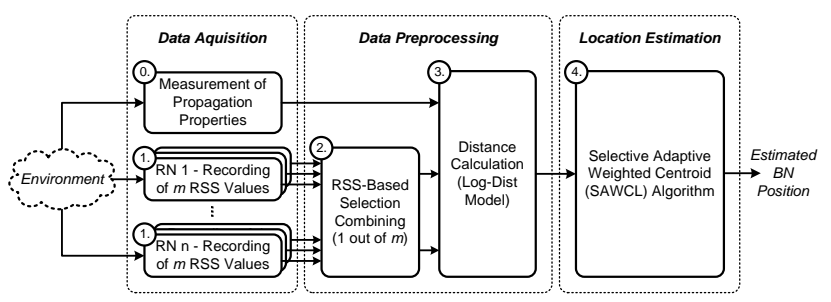

Fig. 3 - Structure of the RSS-based localization system. Source: Own elaboration

When designing an RSS-based indoor locationsensing system it is recommended to concentrate on the proper acquisition of the RSS values since their interpretation has the main influence on the system's accuracy. For an obstructed Indoor environment like the longwall mining application illustrated in Fig. 2 multipath effects have to be taken into account at the propagation of electromagnetic waves. Macroscopic objects with metallic surfaces (e.g. hydraulic shields, shearer, conveyor belt) are characteristic for these environments. Therefore, not only LOS connections but also NLOS connections between transmitter (BN) and receiver (RN) have to be considered. As a consequence of the multipath propagation by reflection, diffraction and scattering effects interferences of different multipath components at the receiver occur. As represented in Fig. 4, the distance-dependent path loss at the propagation of electromagnetic waves between transmitter and receiver is influenced by two different fading components.

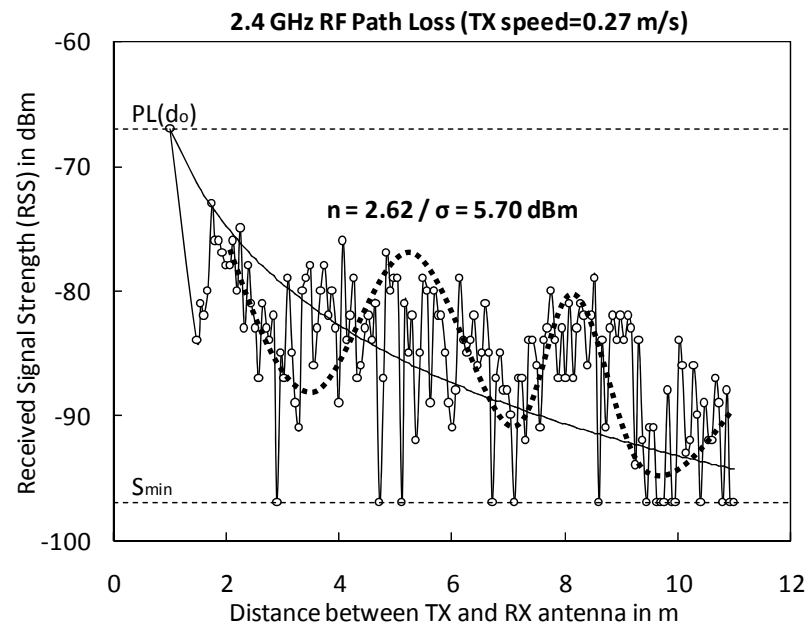

Fig. 4 - 2.4 GHz path loss measurement over a distance of $11 \mathrm{~m}(\mathrm{TX}$ speed $=0.27 \mathrm{~m} / \mathrm{s}, \mathrm{f}=2440.22$ MHz, 250 kHz RF bandwidth, 150 RSS samples, 5 Hz update rate). Source: Own elaboration

Without any disturbances (free space propagation) the distance-depending path loss shows a logarithmic dropping of power with linear increasing distance according to the log-distance path loss model. With (1) the average path loss
$\overline{P L}(d)$ (in $d B m$ ) over a distance $d$ is given by the average path loss over a reference distance $d_{0}$ and the environment-specific propagation coefficient $n$ [4].

$$
\overline{P L}(d)=\overline{P L}\left(d_{0}\right)+10 \cdot n \cdot \log \left(\frac{d}{d_{0}}\right)
$$

For obstructed Indoor environments a lognormally distributed random value $\mathrm{X}_{\sigma}$ can be added to the above equation to take the large-scale fading due to shadowing through obstacles into account. The small-scale fading due to interferences of different multipath components is the main source of errors for the radio-based localization system. Frequency specific signal dropouts in case of destructive interference effects lead to bad distance approximations and a large location estimation error (LEE). In some cases - when the signal strength at the receiver is below the receiver's sensitivity threshold the signal gets lost and no information for the distance between transmitter and receiver can be calculated. Thus, the small-scale fading influences not only the accuracy of the location estimation but also the availability of the system.

A diversity concept with the use of space and frequency diversity at the same time is used to improve the accuracy of the distance estimation and increase the system's availability.

The RF communication takes place by proprietary radio modules from Amber Wireless. Both the $\mathrm{BN}$ and the $\mathrm{RN}$ have a multichannel transceiver with four RF modules which are arranged in a rectangle (from the top view, cf. Fig. 5). Two different frequency bands $(868 \mathrm{MHz}$ and $2.4 \mathrm{GHz}$ ) are used, whereby the diagonal arranged modules use the same frequency band.

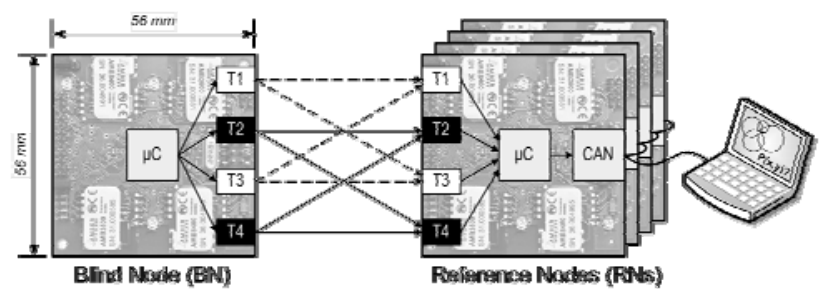

Fig. 5 - Overview of the radio positioning system and diversity concept with eight RF communication channels. Source: Own elaboration

The AMB2500 (T1 and T3) operates at the license-free ISM band at $2.4 \mathrm{GHz} \quad\left(f_{m}=\right.$ $2440.2 \mathrm{GHz}$ ) and is attached by the CC2500 Lowpower RF IC. The AMB8400 (T2 and T4) operates at the license-free ISM band at $868 \mathrm{MHz}\left(f_{m}=\right.$ $868.3 \mathrm{MHz}$ ) and uses the CC1101 Low-power RF IC. For both ICs the MSK modulation, a data rate of $250 \mathrm{kbps}$ and an output power of $+10 \mathrm{dBm}$ are used. Beside space diversity also frequency diversity 
is realized with eight uncorrelated communication channels. In the best case eight RSS values are collected for each RN and the best of them is used for the distance approximation after a selection combining (SC). We use two single SC blocks for each frequency and a following SC for their output as it is shown in Fig. 6. After all the maximum RSS value is forwarded to the distance calculation block.

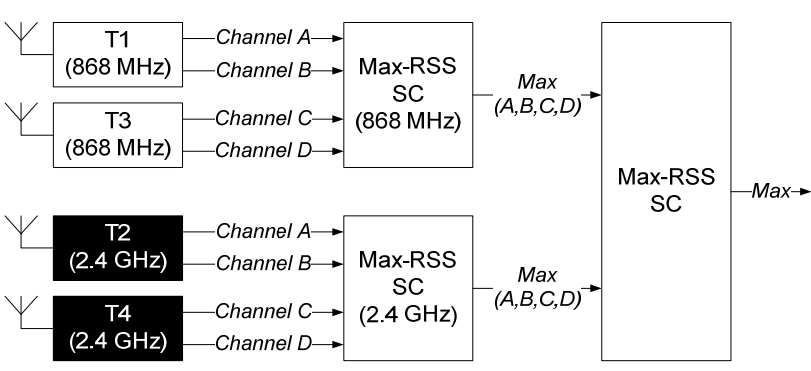

Fig. 6 - Two-stage selection combining (SC) using spatial and frequency diversity channels. Source: Own elaboration

\section{INERTIAL NAVIGATION SYSTEM}

The fundamentals about inertial navigation are given in [12] and [13]. In principle, an INS consists of the inertial sensor platform (inertial measurement unit - IMU) for the acquisition of the motion and the navigation computer for the calculation of the position of the platform. When the sensors are mounted directly to the the body of the platform it is called a strapdown IMU and a strapdown mechanization can be used to compute the position of the platform in the navigation frame.

The strapdown mechanization of the INS is shown in Fig. 7. It uses a three-dimensional navigation frame with six degrees of freedom. The three translatory motions $\left(a_{x}, a_{y}\right.$ and $\left.a_{z}\right)$ and the three rotary motions $\left(w_{x}, w_{y}\right.$ and $\left.w_{z}\right)$ are measured in the body frame. For the translatory motions acceleration sensors are used. The rotary motions are measured with angular rate sensors (gyroscopic sensors).

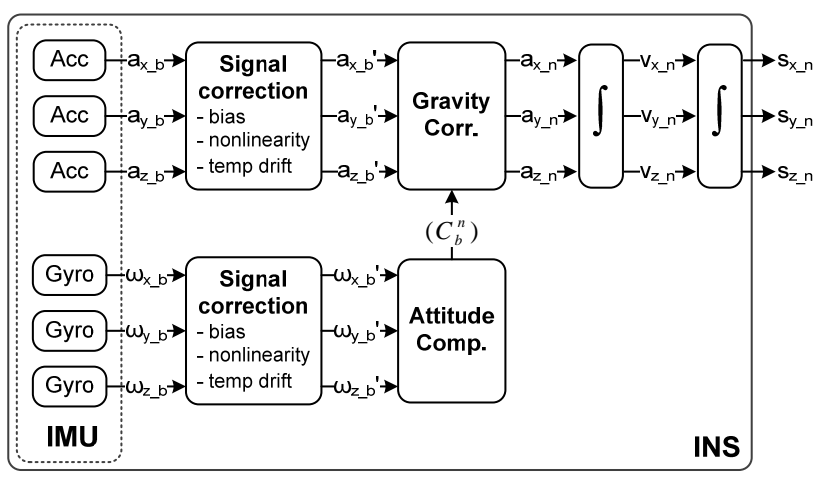

Fig. 7 - Three-dimensional strapdown inertial navigation system. Source: Own elaboration

A temperature compensation is used for the sensors and the values are corrected according to bias and nonlinearity errors. The filtered vector of the angular rates $\vec{w}^{\prime}$ is used to update the attitude of the sensor platform in the navigation frame. The integrated values are expressed by the Euler angles $\phi, \theta$ and $\psi$. For small angular rate changes $\Delta \vec{w}^{\prime}$ the corresponding rotation matrix $C_{b}^{n}$ is given with

$$
C_{b}^{n}=\left[\begin{array}{ccc}
1 & -\psi & \sin \theta \\
\psi & 1 & -\phi \\
-\sin \theta & \phi & 1
\end{array}\right]
$$

$C_{b}^{n}$ is used for the transformation of coordinates from the body to the navigation frame and the gravity correction of the measured accelerations. The corrected acceleration vector in the navigation frame is given with

$$
\vec{a}_{n}=C_{b}^{n} \vec{a}_{b}-\vec{g}
$$

To get the corresponding velocity vector $\vec{v}_{n}$ for the movement between the actual measurement at time step $t$ and the last measurement at time step $t-1$ (3) needs to be integrated.

$$
\vec{v}_{n}(t)=\vec{v}_{n}(t-1)+\int_{t-1}^{t} \vec{a}_{n}(t) d t
$$

The position vector can be calculated by integrating (4) and is given with

$$
\vec{s}_{n}(t)=\vec{s}_{n}(t-1)+\int_{t-1}^{t} \vec{v}_{n}(t) d t .
$$

The Low-cost IMU hardware has six degrees of freedom (DOF) and is shown in Fig. 8. The system architecture has two sockets for the connection of the IMU to the system controller on our multichannel transceiver platform (cf. Fig. 2). We use the features of the IMU platform on both the BN and the RN.

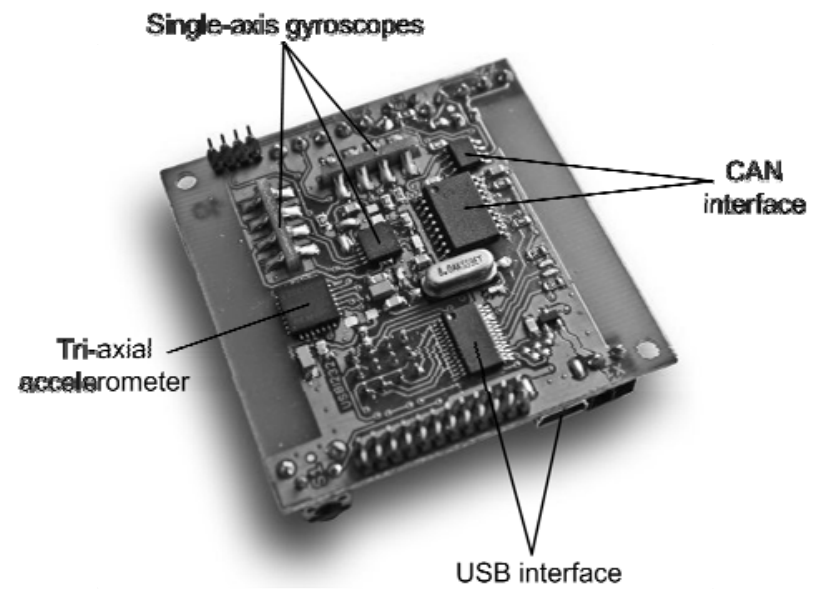

Fig. 8 - Six DOF low-cost IMU hardware platform. Source: Own elaboration 
The USB interface is used for the sensor calibration on the BN and the CAN interface for the connection of the RNs to the data concentrator PC as described in section II.

The $\mathrm{x}-, \mathrm{y}$ - and $\mathrm{z}$-component of the BN's acceleration is measured with a digital LIS3LV triaxial acceleration sensor which operates at a full scale range of $\pm 2 g$. The angular rates around the three axis are measured with analog LY530ALH gyroscopic sensors with a measurement range of $\pm 300 \% \mathrm{sec}$. The actualization rates of all sensors are set to $40 \mathrm{~Hz}$. The detailed sensor characteristics are summarized in Table I.

Table 1. IMU sensor characteristics

\begin{tabular}{|l|c|c|}
\hline \multicolumn{1}{|c|}{ Parameter } & LIS3LV Acc & LY530ALH Gyro \\
\hline $\begin{array}{l}\text { Measurement } \\
\text { range }\end{array}$ & $\pm 2 g$ & $\pm 300 \%$ sec \\
\hline Resolution & $12 \mathrm{Bit}$ & $12 \mathrm{Bit}$ \\
\hline Data rate & $40 \mathrm{~Hz}$ & $40 \mathrm{~Hz}$ \\
\hline $\begin{array}{l}\text { Temperature } \\
\text { sensitivity }\end{array}$ & $0.025 \% /{ }^{\circ} \mathrm{C}$ & $0.05^{\circ} / \mathrm{sec} /{ }^{\circ} \mathrm{C}$ \\
\hline Nonlinearity & $\pm 2 \% \mathrm{FS}$ & $\pm 1 \% \mathrm{FS}$ \\
\hline
\end{tabular}

The sensors need to be calibrated before they can provide useful results. We use an in-field calibration without the need of external equipment (e.g. threeaxis turn-table) as it is proposed in [14].

\section{SENSOR FUSION ALGORITHM}

In [11] a plausibility filtering of the calculated distances between the $\mathrm{BN}$ and the RNs is proposed. In [10] the RSS-based BN position (and not its distances to RNs) is filtered according to an onedimensional motion vector where the INS position acts as a threshold value. This feedforward architecture is easy to implement and requires only a small amount of additional processing steps. Our new approach uses a feedback architecture for the adaption of the estimated position (cf. Fig. 9).

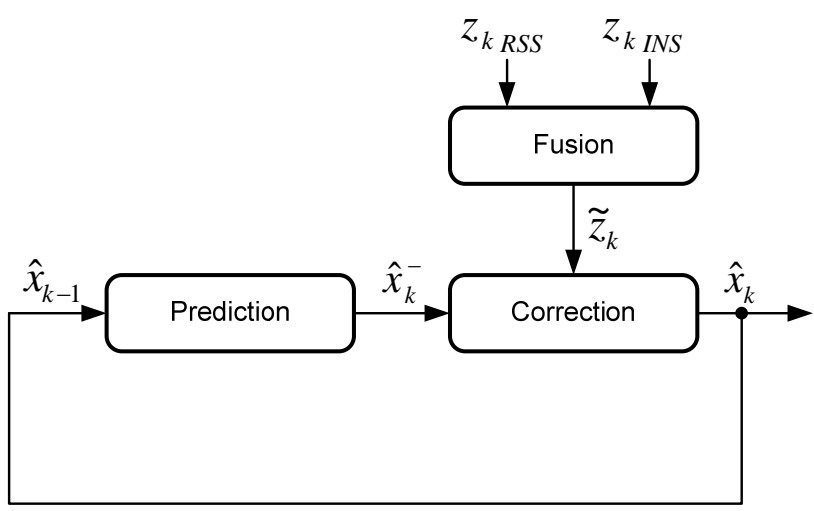

Fig. 9 - Multi-sensor multi-temporal centralized measurement fusion. Source: [15]

The measurements from the RSS-based localization system and the sensor values of the INS are combined with a centralized measurement fusion according to [15]. The resulting observation $\tilde{\mathbf{z}}_{\mathbf{k}}$ is used for the correction of the a priori state estimate $\widehat{x}_{k}^{-}$.

The Kalman filter uses a set of mathematical equations that provides an efficient technique to estimate the state of a discrete-time controlled process. The recursive algorithm predicts the actual process state with the last state and the actual measurement for process input. The model for the process and measurement is given as follows:

$$
\begin{aligned}
& x_{k}=A x_{k-1}+w_{k}, \\
& z_{k}=H_{k} x_{k}+v_{k} .
\end{aligned}
$$

The process noise $\mathbf{w}_{\mathbf{k}}$ and the measurement noise $\mathbf{v}_{\mathbf{k}}$ are assumed to be independent (of each other), white, and with normal probability distributions. We take the covariances of a real life path loss measurement for the initial noise estimations. The filter is divided into two parts, the time update (prediction stage) and the measurement update (correction stage). The time update equations for the filter are the following [16]:

$$
\begin{gathered}
\hat{\mathbf{x}}_{\mathbf{k}}=\mathbf{A} \hat{\mathbf{x}}_{\mathbf{k}-1}^{-}, \\
\mathbf{P}_{\mathbf{k}}^{-}=\mathbf{A P}_{\mathbf{k}-\mathbf{1}} \mathbf{A}^{\mathbf{T}}+\mathbf{Q} .
\end{gathered}
$$

The time update equations project forward the current state $\hat{\mathbf{x}}_{\mathbf{k}}^{-}$and the error covariance estimates $\mathbf{P}_{\mathbf{k}-\mathbf{1}}$ to obtain an a priori state estimate. $\mathbf{Q}$ is the process noise covariance matrix. The $\mathbf{n} \mathbf{x} \mathbf{n}$ matrix $\mathbf{A}$ defines the transition from the state at the previous time step $\mathbf{k}-\mathbf{1}$ to the state at the current step $\mathbf{k}$. The measurement update can be described as follows:

$$
\begin{gathered}
\mathbf{K}_{\mathbf{k}}=\mathbf{P}_{\mathbf{k}}^{-} \mathbf{H}^{\mathrm{T}}\left(\mathbf{H} \mathbf{P}_{\mathbf{k}}^{-} \mathbf{H}^{\mathbf{T}}+\mathbf{R}\right)^{-\mathbf{1}}, \\
\hat{\mathbf{x}}_{\mathbf{k}}=\hat{\mathbf{x}}_{\mathbf{k}}^{-}+\mathbf{K}_{\mathbf{k}}\left(\mathbf{z}_{\mathbf{k}}-\mathbf{H} \hat{\mathbf{x}}_{\mathbf{k}}^{-}\right), \\
\mathbf{P}_{\mathbf{k}}=\left(\mathbf{I}-\mathbf{K}_{\mathbf{k}} \mathbf{H}\right) \mathbf{P}_{\mathbf{k}}^{-} .
\end{gathered}
$$

The Kalman gain $\mathbf{K}_{\mathbf{k}}$ is used to weight the difference between the a priori state estimate $\widehat{\mathbf{x}}_{\mathbf{k}}^{-}$and the current measurement observations $\mathbf{z}_{\mathbf{k}}$. The measurement noise covariance matrix $\mathbf{R}$ is dependent on the variance of the measurement values. The $\mathbf{n} \mathbf{x} \mathbf{n}$ matrix $\mathbf{H}$ relates the state estimate $\widehat{\mathbf{x}}_{\mathbf{k}}^{-}$to the measurement $\mathbf{z}_{\mathbf{k}}$.

\section{EXPERIMENTAL RESULTS}

The test bed for the localization system is a tracking application on the motion test track shown in Fig. 10. Seven RNs are evenly distributed next to the track. Reflecting walls are installed to force multipath RF propagation for a realistic scenario. During the experiment the BN moves between the end positions $\mathrm{A}$ and $\mathrm{B}$ and the RSS and INS measurements are collected on the data concentrator. 


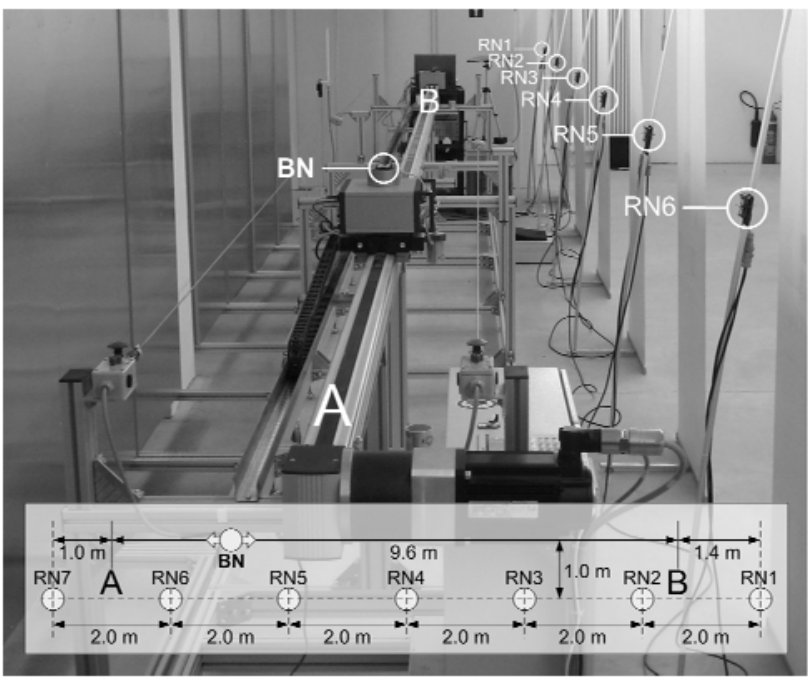

Fig. 10 - RF positioning system on a motion test track in a multipath indoor environment (BN - blind node, $\mathrm{RN}$ - reference node). Source: Own elaboration

A Java application (cf. Fig. 11) is running on the PC for the computation and visualization of the BN's position. The central component of the software is the radio map where the infrastructure is visualized. The circles around the RNs represent the RSS-based distance approximations. The recorded RSS values are also saved in a database together with the INS measurements. Therefore an offline calculation of the BN's position is possible and the performance of different measurement noise covariance matrices for the Kalman filter can be compared easily.

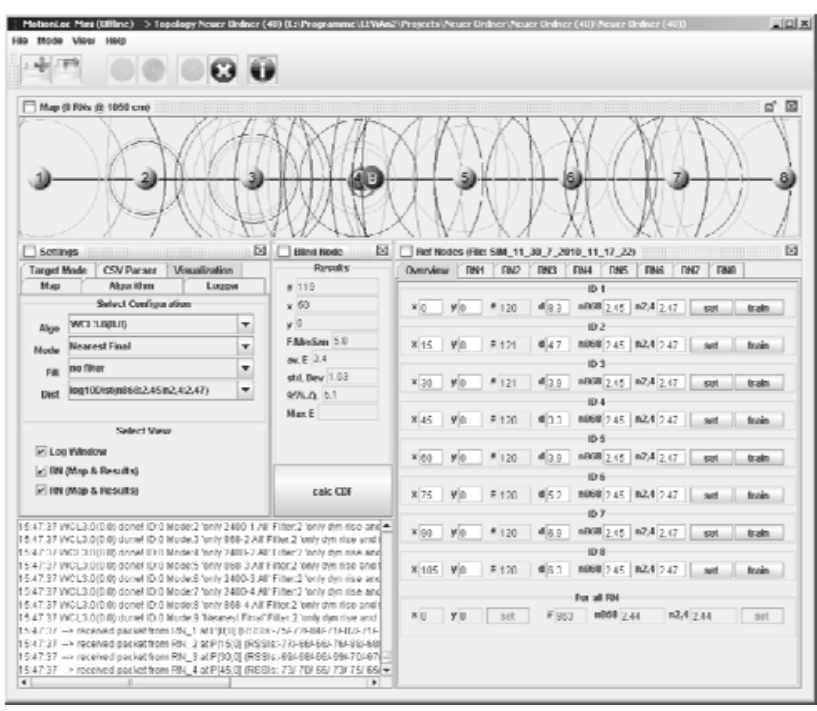

Fig. 11 - Java software platform LEViAn

(Location Estimation with Visualization and Analysis) on the data concentrator PC. Source: Own elaboration

We compare the location estimation error (LEE) of four different configurations. The first configuration is the "RSS" reference localization without any additional filtering. The second configuration "RSS + d_filter" uses the distance filter technique from [11]. The third configuration
"RSS+INS_filter" uses the motion data from an INS to filter the BN position [10]. The fourth configuration "RSS+INS_fusion" is the proposed sensor fusion of the RSS localization and the INS using a Kalman filter. The cumulative distribution functions (CDFs) of all configurations are shown in Fig. 12.

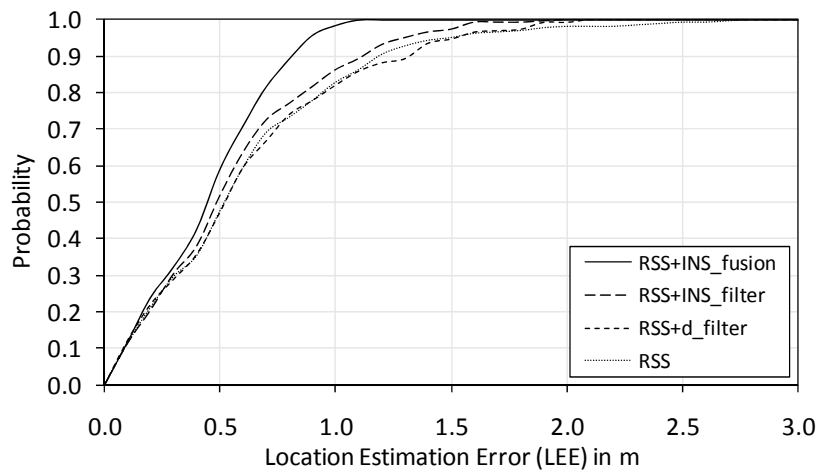

Fig. 12 - Error probability for the location estimation of various filter configurations. Source: Own calculation

A detailed error statistic can be found in Table II. The median error LEEmed is nearly the same for all configurations. Looking at the $\mathbf{9 9}^{\text {th }}$ percentile the influence of the filter and fusion algorithms gets obvious. The maximum error of the RSS localization system without any filter is $\mathbf{2 . 7 8} \mathbf{m}$. With the plausibility filtering of distances the maximum error is reduced by more than $25 \%$ to $2.08 \mathrm{~m}$. With the sensor fusion of the RSS localization and the INS the maximum error is reduced by even more than $62 \%$ to $1.08 \mathrm{~m}$.

Table 2. Performance comparison of different filter configurations (LEE - location estimation error in $\mathbf{m}$ )

\begin{tabular}{|c|c|c|c|c|}
\hline & RSS & $\begin{array}{c}\text { RSS+ } \\
\text { d_filt }\end{array}$ & $\begin{array}{c}\text { RSS+ } \\
\text { INS_filter }\end{array}$ & $\begin{array}{c}\text { RSS+ } \\
\text { INS_fusion }\end{array}$ \\
\hline$L E E_{a v}$ & 0.52 & 0.54 & 0.48 & 0.46 \\
\hline$\sigma_{L E E}$ & 0.49 & 0.46 & 0.39 & 0.26 \\
\hline$L E E_{99 \%}$ & 2.38 & 1.89 & 1.58 & 1.04 \\
\hline$L E E_{\max }$ & 2.78 & 2.08 & 1.93 & 1.08 \\
\hline
\end{tabular}

\section{CONCLUSION AND FUTURE WORK}

Looking at the dimension of the test track with a length of $9.60 \mathrm{~m}$, the error range of the RSS localization without filter covers more than the half of the whole track, which indicates a bad system performance. The proposed sensor fusion is useful to reduce the error in a significant way. The combined system has a sufficient precision and enables a localization for many applications, e.g. for the monitoring of maintenance staff in a longwall mining infrastructure. Since the inertial sensor technology based on MEMS techniques evolves rapidly, the accuracy of the combined system can be 
even more improved with a newer and more precise sensor platform. The purpose of a low-cost localization system should be considered when selecting the appropriate sensors. For the RSS localization system a further development should comprise the replacement of the proprietary RF transceivers by standardized low-power protocols like ZigBee or Bluetooth low energy. For the future we are also consider to test the system in a real scenario of an underground longwall mining application. Therefore, we are currently working on the intrinsic safety of the infrastructure components.

\section{REFERENCES}

[1] I. Guvenc, Enhancements to RSS based indoor tracking systems using Kalman filters, In GSPX \& International Signal Processing Conference, 2003.

[2] E. Elnahrawy, X. Li, and R. Martin, The limits of localization using signal strength: A comparative study, In IEEE Sensor and Ad Hoc Communications and Networks (SECON), 2004, pp. 406-414.

[3] K. Whitehouse, C. Karlof, and D. Culler, A practical evaluation of radio signal strength for ranging-based localization, SIGMOBILE Mobile Computing and Communications Review, (11), (January 2007), pp. 41-52.

[4] T. S. Rappaport, Wireless Communications Principles and Practice, Prentice Hall PTR, 2002.

[5] S. Haykin and M. Moher, Modern Wireless Communications, Pearson Prentice Hall, 2005.

[6] A. Molisch, Wireless Communications, John Wiley \& Sons, 2005.

[7] F. Evennou and F. Marx, Advanced integration of WiFi and inertial navigation systems for indoor mobile positioning, EURASIP Journal on Applied Signal Processing, (2006), (January 2006), pp. 164-164.

[8] H. Wang, H. Lenz, A. Szabo, J. Bamberger and U. Hanebeck, WLAN-based pedestrian tracking using particle filters and lowcost MEMS sensors, In 4th IEEE Workshop on Positioning, Navigation and Communication (WPNC), 2007, pp. 1-7.

[9] P. Coronel, S. Furrer, W. Schott, and B. Weiss, Indoor location tracking using inertial navigation sensors and radio beacons, The
Internet of Things, 2008, pp. 325-340.

[10] A. Fink, H. Beikirch, M. Voss, and C. Schroeder, RSSI-based indoor positioning using diversity and inertial navigation, In IEEE International Conference on Indoor Positioning and Indoor Navigation (IPIN), 2010, pp. 1-7.

[11] A. Fink, H. Beikirch, and M. Voss, Improved indoor localization with diversity and filtering based on received signal strength measurements, International Journal of Computing, (9) 1 (2010), pp. 9-15.

[12] D. Titterton and J. Weston, Strapdown Inertial Navigation Technology, Peter Peregrinus Ltd., 1997.

[13] A. Lawrence, Modern Inertial Technology Navigation, Guidance and Control, SpringerVerlag New York, Inc., 1998.

[14] W. Fong, S. Ong, and A. Nee, Methods for infield user calibration of an inertial measurement unit without external equipment, Measurement Science and Technology, (19) 8 (2008).

[15] H. Mitchell, Multi-Sensor Data Fusion, Springer, 2007.

[16] G. Welch and G. Bishop, An Introduction to the Kalman filter, Chapel Hill, NC, USA, Tech. Rep., 1995.

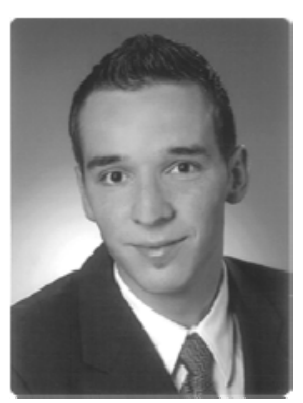

Andreas Fink, received his M.Sc. degree in information technology from the University of Rostock in 2008. Currently he works as a research scientist at the Department of Computer Science and Electrical Engineering. His primary research interests are powerline communication interfaces, wireless communication systems and indoor localization.

Helmut Beikirch, to hold since 1998 the professorship „Electronic Devices and Circuit Design" of the Faculty of Computer Science and Electrical Engineering at the University of Rostock. $\mathrm{He}$ is the Head of the research group in electronic circuit design and communication systems in industrial automation. 